11

\title{
Волновая природа поперечного эффекта Доплера и аномалии „красного“ и „синего“ смещения частоты электромагнитной волны движущегося источника излучения
}

\author{
(C) А.В. Куклин, В.А. Куклин
}

Казанский национальный исследовательский технический университет им. А.Н. Туполева-КАИ. Институт радиоэлектроники и телекоммуникаций,

420111 Казань, Россия

e-mail: iamkvova@gmail.com

(Поступило в Редакцию 31 марта 2018 г.)

\begin{abstract}
Проанализировано электромагнитное поле движущегося с постоянной скоростью гармонического осциллятора. Получено выражение для расчета доплеровского сдвига частоты при релятивистских скоростях его движения в рамках классической электромагнитной теории. Показано, что поперечный эффект Доплера является частным случаем, и в рамках волновой теории он также отличен от нуля. С ростом скорости движения источника излучения растет превышение „красного“ смещения частоты электромагнитной волны над „синим“. Продемонстрирована непротиворечивость предлагаемой модели широко известным экспериментальным данным.
\end{abstract}

DOI: $10.21883 /$ JTF.2019.01.46975.135-18

Появление статьи „Доплера эффект“ в физическом энциклопедическом словаре можно считать результатом сформированной на данный момент времени парадигмы об изменении частоты колебаний, воспринимаемых наблюдателем при относительном движении источника излучения. В соответствии с [1] эффект Доплера „имеет чисто кинематическое происхождение“ и в однородных средах определяется только относительной скоростью движения, углом $\alpha$ между вектором скорости и волновым вектором, а также учитывает влияние $\gamma$-фактора, отражающего релятивистское замедление времени. При этом подчеркивается наличие поперечного эффекта Доплера, который „не имеет никакой волновой специфики“ [1]. При низких скоростях движения ( $\beta$ не более 0.005$)$ источника излучения данная модель описания физического явления дает результаты, соответствующие экспериментальным данным [2-4]. Однако первые результаты экспериментов прямых измерений длины волны движущихся источников излучения с повышенными скоростями ( $\beta$ до 0.005 ) показали необъяснимые отклонения от теоретических расчетов [5]. В работе [6] для объяснения расхождения экспериментальных и расчетных данных обращено внимание на правильность выбора угла $\alpha$ и предложен интересный подход с учетом аберрационного эффекта. Но так как в [5] регистрировался спектр исследуемого излучения, a не изображение самого излучающего объекта $\mathrm{H}_{2}^{+}$, то использование аберрационной поправки считаем не корректным, поскольку она показывает только угловую поправку для определения расположения движущегося объекта в момент его регистрации наблюдателем. К сожалению, других обоснований отклонения расчетных данных нет. В связи с этим задача поиска причины расхождения уникальных экспериментальных данных [5] с существующей моделью, на наш взгляд, является актуальной.

В настоящей работе предлагается метод оценки доплеровского сдвига частоты, основанный на учете только волновых процессов, происходящих при распространении электромагнитной волны в рамках классической электромагнитной теории. Для этого сделана попытка исследования процесса формирования в точке наблюдения электромагнитного поля, создаваемого движущимся с постоянной скоростью простейшим источником излучения.

Объектом исследования в настоящей работе рассматривается гармонический осциллятор, когда на колебания заряда накладывается равномерное (неускоренное) его перемещение с постоянной скоростью $U_{\text {const }}$. Данный объект соответствует всем критериям точечного заряда и запаздывающие потенциалы Лиенара-Вихерта позволяют вычислить векторы напряженности электрического и магнитного полей в произвольной точке [7]. Без ущерба для обобщения выберем систему координат так, чтобы вектор равномерного движения заряда был направлен вдоль координаты $Z$. Направление наблюдения доплеровского сдвига частоты расположим в плоскости $X Z$ под углом $\alpha$ к оси $Z$. Для упрощения расчетных выражений вектор скорости колебаний заряда также расположим в плоскости $X Z$ и перпендикулярно направлению наблюдения, чтобы обеспечить максимум диаграммы направленности излучения осциллятора в этом направлении. Линейные размеры осциллятора существенно меньше расстояний до точки наблюдения и точки расчета компонентов поля, поэтому перемещением заряда за счет колебательного движения при оценке радиуса вектора его положения пренебрегаем. Тогда для вектора скорости движения $\mathbf{U}(t)$ точечного заряда и век- 
тора $\mathbf{R}$, проведенного из точки мгновенного расположения заряда в точку расчета напряженности компонентов поля, можно записать следующие выражения

$$
\begin{gathered}
\mathbf{R}=\left\{x, y, z-U_{\text {const }} t^{\prime}\right\} \\
R=\sqrt{\left|\mathbf{R}_{t^{\prime}=0}\right|^{2}+\left(U_{\text {const }} t^{\prime}\right)^{2}-2\left|\mathbf{R}_{t=0}\right| U_{\text {const }} t^{\prime} \cos [\theta]} \\
U\left(t^{\prime}\right)=\left\{-U_{0} \sin \left[\omega^{\prime} t^{\prime}\right] \cos [\alpha], 0, U_{0} \sin \left[\omega^{\prime} t^{\prime}\right] \sin [\alpha]+U_{\text {const }}\right\},
\end{gathered}
$$

где $\mathbf{R}_{t^{\prime}=0}=\mathbf{r}$ - радиус вектор точки наблюдения в момент начала движения заряда $e_{0}, \theta-$ полярная координата точки расчета напряженности компонентов поля, $\omega^{\prime}-$ круговая частота колебаний заряда осциллятора. В момент времени $t^{\prime}$ определяется состояние движения заряда гармонического осциллятора, предшествующее возмущению искомого поля в точке наблюдения. При данных допущениях, используя общепринятую методику расчетов [7], получим выражение для напряженности электрического поля, создаваемого рассматриваемым гармоническим осциллятором в дальней зоне в точке наблюдения доплеровского сдвига частоты

$$
\begin{aligned}
\mathbf{E}= & \left\{-\frac{U_{0} \omega^{\prime} e_{0}}{c^{2} r} \sin (\theta) \frac{\frac{\beta c t^{\prime}}{r} A}{B} \cos \left(\omega^{\prime} t^{\prime}\right),\right. \\
& \left.\frac{U_{0} \omega^{\prime} e_{0}}{c^{2} r} \frac{\left[1-\frac{\beta c t^{\prime}}{r} \cos (\theta)\right] A}{B} \cos \left(\omega^{\prime} t^{\prime}\right), 0\right\},
\end{aligned}
$$

где использованы обозначения

$$
\beta=\frac{U_{\mathrm{const}}}{c}
$$

$A=\left\{\cos (\alpha-\theta)-\beta \cos (\alpha) \sqrt{1+\frac{(\beta c)^{2} t^{\prime 2}}{r^{2}}-2 \frac{\beta c t^{\prime}}{r} \cos (\theta)}\right\}$,

$B=\left\{\beta\left[\frac{\beta c t^{\prime}}{r}-\cos (\theta)\right]+\sqrt{1+\frac{(\beta c)^{2} t^{\prime 2}}{r^{2}}-2 \frac{\beta c t^{\prime}}{r} \cos (\theta)}\right\}^{3}$.

Выражение для напряженности магнитного поля не несет какой-либо дополнительной информации, поэтому не приводится.

Формулы выражения (2) показывают наличие радиальной составляющей напряженности электрического поля, изменяющейся как $1 / r$, даже в дальней зоне. В силу этого наряду с традиционной радиальной составляющей электромагнитного поля в дальней зоне должна наблюдаться и тангенциальная составляющая электромагнитной волны вдоль полярной координаты $\theta$. Очевидно, что изменение структуры компонентов поля должно повлиять на характер распространения, создаваемой электромагнитной волны, что показывают результаты расчетов, приведенных на рис. 1. Диаграммы направленности излучения осциллятора рассчитаны для различных скоростей поступательного движения осциллятора $\beta$ и нормированы коэффициентом

$$
U_{0}^{2} \omega^{2} / 2 c^{4} r^{2}
$$

Важно отметить, что при выбранном направлении наблюдения $\alpha=1.344\left(77^{\circ}\right.$ ) (по условиям проведения экспериментов в [5]) для покоящегося точечного источника излучения с увеличением скорости происходит смещение направления распространения электромагнитной волны в сторону направления движения осциллятора.

Фазовый множитель $\cos \left(\omega^{\prime} t^{\prime}\right)$ выражения (2) определяет колебательный процесс гармонического осциллятора, который является причиной изменений компонентов электромагнитного поля, достигающих точки наблюдения с некоторой задержкой. Оценим величину этой задержки для моментов времени, отстоящих друг от друга на величину периода колебаний заряда $\tau^{\prime}$.

Состояние осциллятора в момент времени $t^{\prime}$ приведет к формированию электромагнитного поля в точке наблюдения с некоторой задержкой, определяемой расстоянием от точки текущего положения заряда до точки наблюдения. Этот момент времени можно определить следующим выражением:

$$
t=t^{\prime}+\frac{\sqrt{r^{2}+\left(\beta c t^{\prime}\right)^{2}-2 r \beta c t^{\prime} \cos (\alpha)}}{c} .
$$

К моменту завершения периода колебаний заряда $\tau^{\prime}$ он переместится и изменится расстояние до точки наблюдения. Момент времени прихода электромагнитного излучения из этой точки в точку наблюдения определяется уже следующее образом

$$
\begin{aligned}
& t+\tau=\left(t^{\prime}+\tau^{\prime}\right) \\
& +\frac{\sqrt{r^{2}+\left(\beta c\left(t^{\prime}+\tau^{\prime}\right)\right)^{2}-2 r \beta c\left(t^{\prime}+\tau^{\prime}\right) \cos (\alpha)}}{c} .
\end{aligned}
$$

Очевидно, что периодичность изменения напряженностей электрического и магнитного полей определяется цикличностью колебательных процессов осциллятора. Поэтому можно считать, что величина $\tau$ в выражении (6) отражает период колебательных процессов в точке наблюдения. А следовательно, фазовому множителю $\cos \left(\omega^{\prime} t^{\prime}\right)$ выражения (2) в точке наблюдения электромагнитной волны может быть сопоставлен новый фазовый множитель $\cos \left(\omega t^{\prime}\right)$ с соответствующей заменой $\omega^{\prime}>\omega$ в выражении для амплитуды колебательного процесса.

Физический смысл промежуток времени $\tau$ имеет только при условии, что $t^{\prime} \geq \frac{r}{c}$, и с учетом условия дальней зоны, исходя из (4) и (5), может быть определен следующим выражением:

$$
\tau=\tau^{\prime}\left(1+\frac{\beta\left(\beta c t^{\prime}-r \cos (\alpha)\right)}{\sqrt{r^{2}+\left(\beta c t^{\prime}\right)^{2}-2 r \beta c t^{\prime} \cos (\alpha)}}\right) .
$$




\begin{tabular}{|c|c|c|c|c|}
\hline$\beta \cdot 10^{2}$ & $\begin{array}{c}\Delta \lambda_{\text {к }}, \\
\text { „красное“ смещение, } \\
\text { nm }\end{array}$ & $\begin{array}{c}\Delta \lambda_{\mathrm{c}}, \\
\text { „синее“ } \\
\mathrm{nm}\end{array}$ & $\begin{array}{c}\Delta \lambda_{\text {к }} \text { расчет по }(8) \\
\text { „красное“ смещение, } \\
\text { nm }\end{array}$ & $\begin{array}{l}\Delta \lambda_{\text {c расчет по (8) }} \\
\text { „синее“ смещение, } \\
\text { nm }\end{array}$ \\
\hline $\begin{array}{c}4.618[5] \\
\alpha=1.344\end{array}$ & 7.34 & 5.95 & 7.94 & 5.23 \\
\hline $\begin{array}{l}4.315[5] \\
4.00[5] \\
1.788[5] \\
1.712[5] \\
1.665[5] \\
1.632[5] \\
1.549[5] \\
1.496[5] \\
1.460[5] \\
1.385[5] \\
1.366[5] \\
1.264[5]\end{array}$ & $\begin{array}{l}6.87 \\
6.24 \\
2.4 \\
2.37 \\
2.30 \\
2.16 \\
2.11 \\
2.05 \\
1.97 \\
1.87 \\
1.91 \\
1.72\end{array}$ & $\begin{array}{l}5.75 \\
5.40 \\
2.29 \\
2.28 \\
2.20 \\
2.09 \\
2.05 \\
1.96 \\
1.90 \\
1.81 \\
1.86 \\
1.68\end{array}$ & $\begin{array}{l}7.43 \\
6.81 \\
2.55 \\
2.50 \\
2.42 \\
2.29 \\
2.24 \\
2.14 \\
2.06 \\
1.96 \\
2.0 \\
1.79\end{array}$ & $\begin{array}{l}5.11 \\
4.82 \\
2.14 \\
2.14 \\
2.08 \\
1.95 \\
1.94 \\
1.86 \\
1.79 \\
1.71 \\
1.77 \\
1.59\end{array}$ \\
\hline $\begin{array}{c}0.818[4] \\
\alpha=0.075\end{array}$ & 5.60 & 5.55 & 5.3535 & 5.3530 \\
\hline $\begin{array}{l}0.2888[2] \\
\alpha=0.122\end{array}$ & 1.39 & & 1.39358 & 1.39346 \\
\hline $\begin{array}{l}0.3147 \\
0.3361 \\
0.3806 \\
0.4433\end{array}$ & $\begin{array}{l}1.53 \\
1.64 \\
1.85 \\
2.12\end{array}$ & & $\begin{array}{l}1.51856 \\
1.62183 \\
1.83683 \\
2.13915\end{array}$ & $\begin{array}{l}1.51842 \\
1.62167 \\
1.83637 \\
2.13887\end{array}$ \\
\hline $\begin{array}{c}0.525[3] \\
\alpha=0.122\end{array}$ & 2.582 & & 2.53343 & 2.53303 \\
\hline $\begin{array}{l}0.5313 \\
0.5458 \\
0.5979 \\
0.6569 \\
0.6721\end{array}$ & $\begin{array}{l}2.605 \\
2.653 \\
2.94 \\
3.193 \\
3.25\end{array}$ & & $\begin{array}{l}2.56383 \\
2.63381 \\
2.88524 \\
3.16998 \\
3.24334\end{array}$ & $\begin{array}{l}2.56342 \\
2.63338 \\
2.88473 \\
3.16936 \\
3.24264\end{array}$ \\
\hline
\end{tabular}

Отсюда получаем выражение для сдвига частоты за счет движения гармонического осциллятора с постоянной скоростью $\beta$

$$
\begin{aligned}
& z=\frac{\omega^{\prime}-\omega}{\omega}=\frac{\beta\left(\beta c t^{\prime}-r \cos (\alpha)\right)}{\sqrt{r^{2}+\left(\beta c t^{\prime}\right)^{2}-2 r \beta c t^{\prime} \cos (\alpha)}}, \\
& \lambda=\lambda^{\prime}\left(1+\frac{\beta\left(\beta c t^{\prime}-r \cos (\alpha)\right)}{\sqrt{r^{2}+\left(\beta c t^{\prime}\right)^{2}-2 r \beta c t^{\prime} \cos (\alpha)}}\right) .
\end{aligned}
$$

В начальный момент времени $t^{\prime}=r / c$ (и когда промежуток времени наблюдения за изменением частоты электромагнитного поля значительно меньше $r / c$ ) данная формула упрощается и принимает вид

$$
\begin{aligned}
& z=\frac{\omega^{\prime}-\omega}{\omega}=\frac{\beta(\beta-\cos (\alpha))}{\sqrt{1+\beta^{2}-2 \beta \cos (\alpha)}}, \\
& \lambda=\lambda^{\prime}\left(1+\frac{\beta(\beta-\cos (\alpha))}{\sqrt{1+\beta^{2}-2 \beta \cos (\alpha)}}\right) .
\end{aligned}
$$

Полученное выражение (7) существенно отличается от классической релятивистской формулы, где не учитывается смещение источника излучения относительно наблюдателя, что особенно проявляется при релятивистских скоростях его движения. Это отличие связано с тем, что традиционная модель описания эффекта Доплера ограничена рассмотрением процессов распространения только плоской электромагнитной волны [7]. Поэтому предлагаемое выражение (7) для расчета доплеровского сдвига частоты является более общим и показывает новые стороны проявления рассматриваемого физического явления. Во-первых, оно отражает изменение частоты электромагнитного излучения в течение промежутка времени наблюдения за частотой исследуемого сигнала, что с очевидностью наблюдается для акустических волн. Зависимость доплеровского сдвига частоты от времени связана с изменением положения источника излучения в процессе перемещения относительно точки наблюдения, т.е. связана с изменением угла между вектором скорости и волновым вектором. Этим 


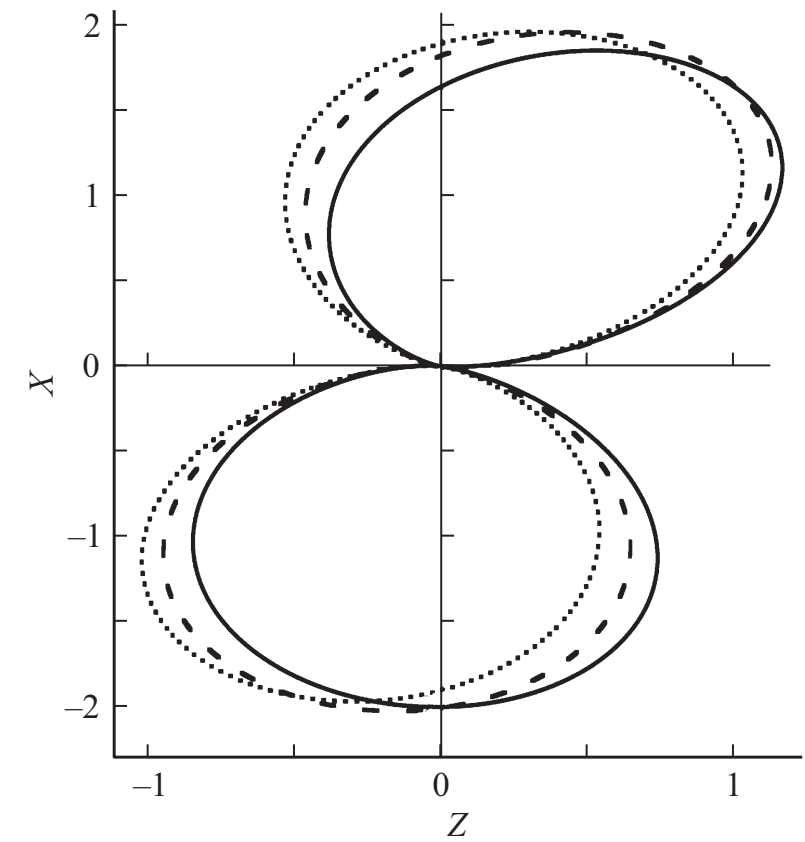

Рис. 1. Нормированная диаграмма направленности рассматриваемого гармонического осциллятора. Направление наблюдения $\alpha=1.344\left(77^{\circ}\right)$. Точки $-\beta=0$, штрихи $-\beta=0.05$, сплошная линия $-\beta=0.1$.

можно пренебречь только на больших расстояния между источником и наблюдателем при условии, если промежуток времени наблюдения существенно меньше $r / c$. Во-вторых, выражение (7) позволяет оценить изменение частоты и для сферической электромагнитной волны на различных расстояниях от точечного источника излучения во всем диапазоне скоростей его движения. В-третьих, в соответствии с (7), как и в рамках релятивисткой теории, поперечный эффект Доплера отличен от нуля. При получении этого выражения использовалась классическая электромагнитная теория формирования и распространения электромагнитной волны, поэтому можно утверждать, что и поперечный эффект Доплера имеет волновую природу в отличие от утверждения [1].

На рис. 2 приведены результаты сравнительных расчетов доплеровского сдвига частоты (длины волны) по классической, релятивистской и предлагаемой в работе модели. Характер зависимостей по релятивистской и предлагаемой модели описания эффекта Доплера практически одинаков, но существенно отличается от классической теории. В области же скоростей до $\beta \sim 0.05$ характер зависимостей всех трех моделей данного физического процесса одинаков и количественное расхождение расчетов незначительно и не превышает 20\%.

Количественное различие предлагаемой модели описания эффекта Доплера от релятивистской, наряду с вышеизложенным обоснованием причины разногласий, безусловно, требуют и экспериментального подтверждения. К сожалению база экспериментальных данных не велика. Поэтому проверим выражение (8) в условиях всех широко известных экспериментов [2-5], несмотря на те или иные вопросы к проведению измерений. В данных работах приводятся экспериментальные данные по измерениям доплеровского сдвига частоты при скоростях излучающих частиц до $\beta \sim 0.05$. Результаты сравнения наших расчетов и экспериментальных данных [2-5] сведены в таблицу.

Анализ сравнения расчетных данных по формуле (8) показывает хорошее совпадение с экспериментальными данными работ [2,3]. Отклонение наших расчетных данных не превышает 1.5\%. Несколько хуже обстоит дело при сравнении результатов экспериментов, приведенных в работах $[4,5]$. В этом случае разница достигает 10-12\%. Установлено, что при измерении доплеровского сдвига частоты в этих работах между излучающими ионами и спектрографом были использованы дополнительные оптические элементы (объективы,
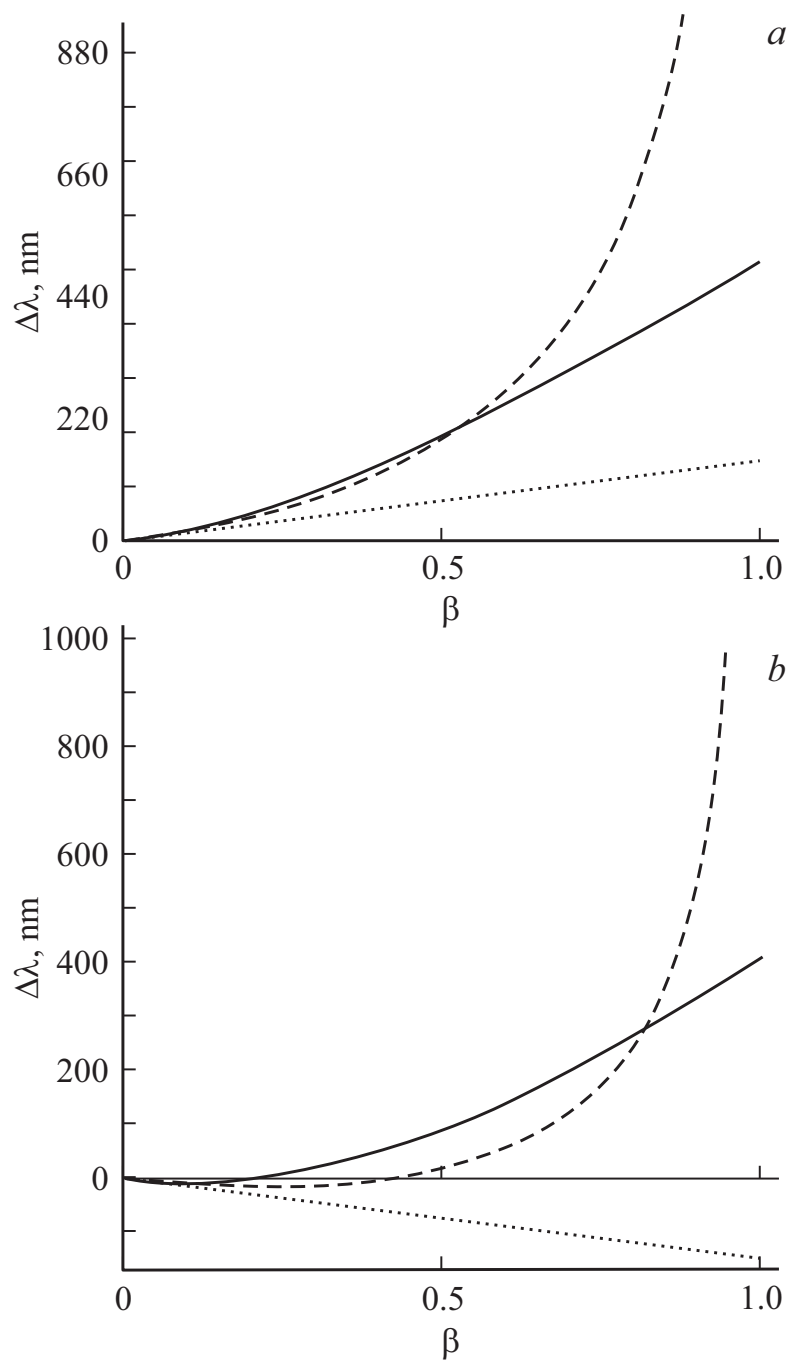

Рис. 2. Сравнительные расчеты доплеровского сдвига длины волны. Направление наблюдения $\alpha=1.344\left(77^{\circ}\right) . a-$ „красное“ смещение; $b-$ „синее“ смещение. Точки - классическая теория; штрихи - релятивистское соотношение; сплошная линия - расчет по предлагаемой модели (8). 
коллиматоры и др.), оказывающие влияние на апертурный угол поля зрения наблюдения. Провести детальную оценку влияния данных элементов на результаты измерений, указанных в работе [4], не представляется возможным в силу отсутствия полных данных. Однако поскольку эксперименты в [4] проводились при углах, близких к $0^{\circ}\left(180^{\circ}\right)$, „неоднозначность“ апертурного угла в меньшей мере влияет на результаты вычислений, чем при углах, близких к $\pi / 2$, которые использовались в [5].

Благодаря подробному описанию условий проведения эксперимента в работе [5] можно сделать некоторые оценки „неоднозначности“ апертурного угла. Конструкция коллиматора состоит из пластин, расстояние между которыми $0.5 \mathrm{~mm}$ при длине $116 \mathrm{~mm}$. Это позволяет дать минимальную оценку апертурного угла объектива $1.04^{\circ}$, с учетом одного отражения от пластин объектива, в пределах которого ослаблением интенсивности излучения можно пренебречь. Лучи, отклоняющиеся от оси на угол $>1.04^{\circ}$ для такой конструкции коллиматора, за счет переотражений будут существенно ослабляться. Экспериментальная оценка апертурного угла на одной щели между медными пластинами дала величину $2.17^{\circ}$ по уровню половинной интенсивности прошедшего через щель света. При такой неопределенности угла наблюдения становится важным критерий выбора величины угла $\alpha$, которую необходимо использовать для расчета сдвига частоты по формуле (8). По условиям экспериментов [5] через использованный объектив на спектрограф должны одновременно проходить лучи от покоящегося и движущегося ионов $\mathrm{H}_{2}^{+}$, разница в направлении которых зависит от скорости $\beta$ (см. рис. 1). За счет диафрагмирования на спектрограф не пройдут лучи, дающие коротковолновую и длинноволновую части спектральных линий „синего“ и „красного“ смещения соответственно. Поэтому микрофотограмма спектра в [5] показала асимметрию спектральных линий от движущихся ионов $\mathrm{H}_{2}^{+}$. Данная асимметрия говорит о том, что смещенные спектральные линии сформированы только той частью точечного источника излучения, направление распространения которого находилось в пределах апертурного угла объектива. В связи с этим максимумам зарегистрированных смещенных спектральных линий соответствуют углы наблюдения $\alpha-1.04$ и $\alpha+1.04$ для „синего“ и „красного“ смещений соответственно. При использовании этих углов расхождение экспериментальных данных [5] с расчетами по (8) уменьшаются до уровня погрешности измерения длины волны.

В работах [2,3] авторами экспериментально не обнаружена аномалия „красного“ и „синего“ доплеровского смещения частоты. Это связано с тем, что при малых скоростях и углах наблюдения, близких к $0^{\circ}\left(180^{\circ}\right)$, аномалия незначительна. При повышении скорости движения источника излучения точность измерения в [4] уже позволила экспериментально обнаружить разницу смещения частоты наблюдаемого излучения при движении „на““ и „от“ наблюдателя. Переход к исследованию доплеровского сдвига частоты при углах, близких к „поперечному“, и дальнейшее увеличение скорости изучаемых объектов показал уверенное различие „красного“ и „синего“ смещения частоты [5]. Расчеты в соответствии с предлагаемой моделью также подтверждают данный экспериментальный факт наличия аномалии „красного“ и „синего“ смещения при релятивистских скоростях.

Таким образом, исследование процесса формирования электромагнитного поля движущегося гармонического осциллятора (точечного источника электромагнитного излучения) показало возможность расчета доплеровского сдвига частоты при релятивистских скоростях его движения, оставаясь в рамках классической электродинамической модели, описывающей волновые процессы распространения электромагнитной энергии. Предлагаемая модель описания данного физического процесса позволяет разрешить существующие на данный момент противоречия между экспериментальными и расчетными данными. Это дает основание утверждать, что и продольный и поперечный эффект Доплера, аномалия „красного“ и „синего“ смещения имеет одну и ту же волновую природу.

Авторы признательны Данилаеву М.П. и Сулимову А.И. за полезные дискуссии в процессе работы над статьей и за живое участие в обсуждении полученных результатов.

\section{Список литературы}

[1] Физика. Большой энциклопедический словарь / Под ред. А.М. Прохорова. М.: Ф50 Большая Российская энциклопедия, 4-е изд. 1998.944 с.

[2] Ives H.E., Stilwell G.R. // J. Opt. Soc. Amer. 1938. Vol. 28. N 7. P. 215-226.

[3] Ives H.E., Stilwell G.R. // J. Opt. Soc. Amer. 1941. Vol. 31. N 5. P. 369-374.

[4] Mandelberg H.I., Witten L. // J. Opt. Soc. Amer. 1962. Vol. 52. N 5. P. 529-536.

[5] Победоносцев Л.А., Крамаровский Я.М., Паршин П.Ф., Селезнев Б.К., Березин Ф.Б. // ЖТФ. 1989. Т. 59. Вып. 3. C. $84-89$.

[6] Беклямишев В.О. // ЖТФ. 1999. Т. 69. Вып. 2. С. 124-126.

[7] Ландау Л.Д., Лифиии Е.M. Теоретическая физика. Т. II. Теория поля. М.: Наука, 1988. 509 с. 